\title{
Overview of ATLAS results
}

Iwona Grabowska-Bold on behalf of the ATLAS Collaboration

AGH University of Science and Technology, Al. Mickiewicza 30, 30-059 Krakow, Poland

\begin{abstract}
The heavy-ion programme in the ATLAS experiment at the Large Hadron Collider aims to probe and characterise hot and dense matter created in relativistic lead-lead collisions. Moreover, smaller collision systems involving nuclei and hadrons are of interest to disentangle initial- from final-state effects. This report presents new results based on lead-lead and proton-proton data collected at $\sqrt{s_{\mathrm{NN}}}=5.02 \mathrm{TeV}$ in 2015, including measurements of bulk collectivity, charged-particle production, electroweak bosons, photon-jet correlations, and quarkonium suppression. First attempts to measure electromagnetic processes in ultra-peripheral collisions are also discussed.
\end{abstract}

Keywords: Quarkonia suppression, Gamma-jet correlations, Light-by-light scattering, Angular correlations

\section{Introduction}

The Quark-Gluon Plasma (QGP) was first observed in experiments at RHIC $[1,2,3,4]$ and since then it has been studied in relativistic heavy-ion collisions. Significantly higher collision energies available at the LHC as compared to RHIC, result in increased volume, lifetime and temperature of the QGP.

The LHC provided lead-lead $(\mathrm{Pb}+\mathrm{Pb})$ collisions at $\sqrt{s_{\mathrm{NN}}}=2.76 \mathrm{TeV}$ in years $2010-2013$ (so-called Run 1) and at $\sqrt{s_{\mathrm{NN}}}=5.02 \mathrm{TeV}$ in Run 2 which commenced in 2015 after a technical break. In addition to the $\mathrm{Pb}+\mathrm{Pb}$ system, proton-lead $(p+\mathrm{Pb})$ and proton-proton $(p p)$ collisions are of interest for the heavy-ion measurements. They serve as reference systems to disentangle initialfrom final-state effects. The ATLAS experiment [5] collected $p+\mathrm{Pb}$ data at $\sqrt{s_{\mathrm{NN}}}=5.02 \mathrm{TeV}$ in 2013 while $p p$ collisions at the same center-of-mass energy were provided prior to the $\mathrm{Pb}+\mathrm{Pb}$ data taking in 2015 .

In the heavy-ion programme at ATLAS two major areas can be identified. A soft sector in which the analyses aim at elucidating long-wavelength properties of the system using tools such as angular correlations. On the other hand, the measurements in the hard sector result in a better understanding of short-range interactions which in turn can be utilised to probe properties of the
QGP. Typical measurements are quenching/suppression of various hard probes.

The ATLAS Collaboration extensively studied various observables at lower Run 1 energy. In this report selected results from the aforementioned two sectors will be presented based on $\mathrm{Pb}+\mathrm{Pb}$ and $p p$ data samples collected at higher center-of-mass energy $\sqrt{s_{\mathrm{NN}}}=5.02 \mathrm{TeV}$ in 2015. In addition, at conditions available in $\mathrm{Pb}+\mathrm{Pb}$ collisions in Run 2 a measurement of light-by-light scattering can be attempted for the first time. Its results will be discussed as well.

\section{Quarkonia production}

ATLAS has reported on measurements of prompt and non-prompt differential production cross sections and nuclear modification factors of the $J / \psi$ and $\psi(2 S)$ mesons [6]. The measurements are performed in the dimuon decay channel in $\mathrm{Pb}+\mathrm{Pb}$ collisions at $\sqrt{s_{\mathrm{NN}}}=5.02 \mathrm{TeV}$, with an integrated luminosity of $0.49 \mathrm{nb}^{-1}$, and in $p p$ collisions at $\sqrt{s}=5.02 \mathrm{TeV}$, with an integrated luminosity of $25 \mathrm{pb}^{-1}$. The measurements are presented for $9<p_{\mathrm{T}}<40 \mathrm{GeV}$ in transverse momentum, and $|y|<2.0$ in rapidity.

The suppression of charmonium states is quantified 
by the nuclear modification factor, $R_{\mathrm{AA}}$, which can be defined for a given centrality class as:

$$
R_{\mathrm{AA}}=\frac{N_{\mathrm{AA}}}{\left\langle T_{\mathrm{AA}}\right\rangle \times \sigma^{p p}},
$$

where $N_{\mathrm{AA}}$ is the per-event yield of charmonium states measured in $\mathrm{Pb}+\mathrm{Pb}$ collisions, $\left\langle T_{\mathrm{AA}}\right\rangle$ is the mean nuclear thickness function and $\sigma^{p p}$ is the cross section for the production of the corresponding charmonium states in $p p$ collisions at the same energy.

A strong suppression of both prompt and non-prompt $J / \psi$ and $\psi(2 S)$ mesons is found. The maximal suppression of both prompt and non-prompt $J / \psi$ is observed for the most central events. The distribution of the dependence of $R_{\mathrm{AA}}$ on centrality is approximately the same for both prompt and non-prompt $J / \psi$. The similarity of this shape for both prompt and non-prompt $J / \psi$ is striking since in the simplest interpretation the observed prompt mesons come predominantly from potentially fragile composite systems exposed to the hot dense medium while the non-prompt mesons come predominantly from $b$-quarks traversing through the medium; thus, one might expect different trends as a function of centrality.

Figures 1 and 2 show ratios of $R_{\mathrm{AA}}$ of $\psi(2 S)$ to $J / \psi$ meson production as a function of the number of participants, $N_{\text {Part }}$, for both prompt and non-prompt components, respectively. Values consistent with unity are measured for the non-prompt mesons, while the values observed for the prompt mesons are below unity. This result is consistent with what would be expected for $b$-quarks in the hot dense medium with the same behaviour for both mesons, while composite mesons formed in the hot and dense medium are affected differently. In particular, the $\psi(2 S)$ meson is suppressed more than the $J / \psi$ meson, a pattern consistent with the lower binding energy of the $\psi(2 S)$ meson causing it to have a lower formation and survival probability in the QGP for the values of $p_{\mathrm{T}}^{\psi(n S)}$ sampled in this measurement.

\section{Photon-jet correlations}

ATLAS has presented a study of photon-jet angular and momentum correlations in $\sqrt{s_{\mathrm{NN}}}=5.02 \mathrm{TeV}{ }_{100}$ $\mathrm{Pb}+\mathrm{Pb}$ and $p p$ events with photons with transverse mo- 101 menta of $60<p_{\mathrm{T}}^{\gamma}<200 \mathrm{GeV}$ [7]. The data are statisti- 102 cally corrected for the presence of combinatoric photon- 103 jet pairs and of dijet pairs where one of the jets has been ${ }_{104}$ misidentified as a photon. The measured quantities in 105 data are compared to the analogous quantities in sim- 106 ulated photon-jet events overlaid with minimum-bias ${ }_{107}$

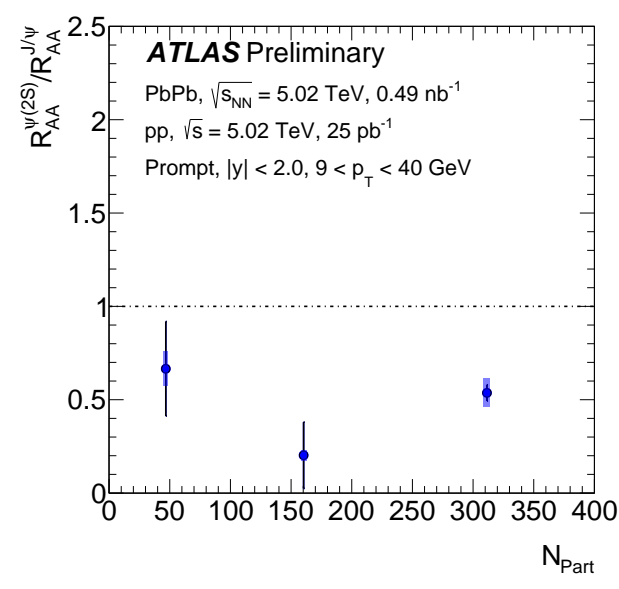

Figure 1: Ratio of $R_{\mathrm{AA}}$ for $\psi(2 S)$ to $J / \psi$ as a function of centrality expressed as $N_{\text {Part }}$, for prompt meson production [6]. The narrow error bar represents the statistical uncertainties while the wider error bar represents the total systematic uncertainty.

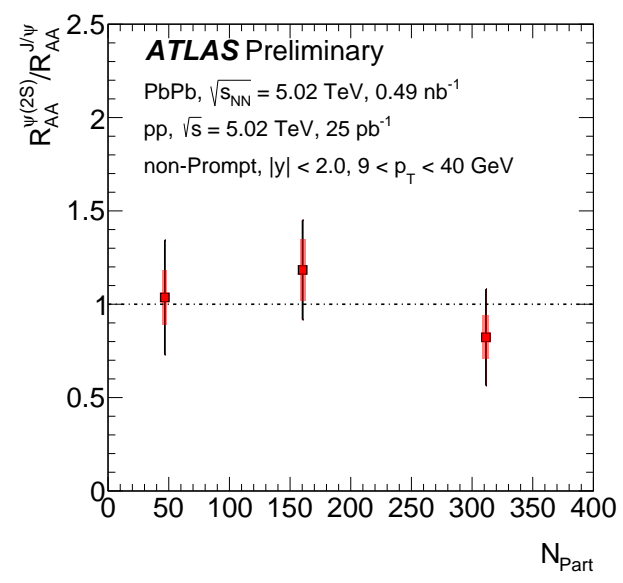

Figure 2: Ratio of $R_{\mathrm{AA}}$ for $\psi(2 S)$ to $J / \psi$ as a function of centrality expressed as $N_{\text {Part }}$, for non-prompt meson production [6]. The narrow error bar represents the statistical uncertainties while the wider error bar represents the total systematic uncertainty. jet events overlaid with minimum-bias $\mathrm{Pb}+\mathrm{Pb}$ data. In tially modified with respect to the results of the simulation, in a manner that is consistent with a shift to lower values of $x_{\mathrm{J} \gamma}$. The distributions have a different shape (they do not exhibit a clear peak within the visible $x_{\mathrm{J} \gamma}$ 
range), and the integrated yield of associated jets above the minimum $x_{\mathrm{J} \gamma}$ value is suppressed. This pattern of modification is indicative of a picture of parton-energy loss in the hot and dense medium.

These modifications are systematic in collision centrality and $p_{\mathrm{T}}^{\gamma}$, consistent with the picture that the partons initially produced opposite to a high- $p_{\mathrm{T}}$ photon lose energy in their interactions with the QGP but remain angularly well-correlated with the photon direction.

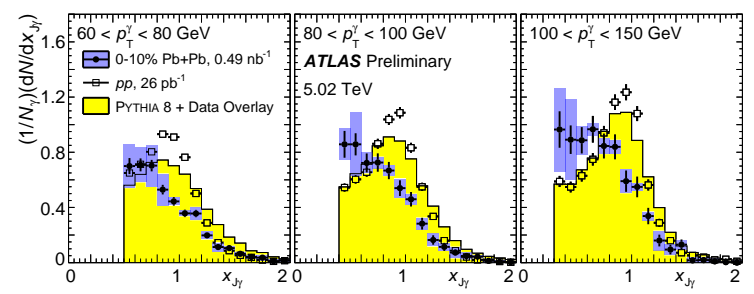

Figure 3: Measured distributions of the jet-to-photon transverse momentum ratio $x_{\mathrm{J} \gamma}$ in $0-10 \% \mathrm{~Pb}+\mathrm{Pb}$ collisions (filled circles), $p p$ collisions (open squares) and PyтнIA8 simulation overlaid with $0-10 \%$ $\mathrm{Pb}+\mathrm{Pb}$ data (yellow histogram) [7]. Each panel shows a different $p_{\mathrm{T}}^{\gamma}$ selection.

\section{Particle production in the $p+\mathrm{Pb}$ system}

\section{Charged-particle spectra}

In Run 1 LHC collaborations performed measurements of inclusive charged particle production $[9,10]$ that in extreme cases showed an enhancement of approximately $30 \%$ with respect to unity in $R_{p \mathrm{~Pb}}$ for particles with $p_{\mathrm{T}}>20 \mathrm{GeV}$. All these measurements used a reference which was derived by interpolating between $p p$ data taken at other $\sqrt{s}$ as no reference data were available at $\sqrt{s}=5.02 \mathrm{TeV}$ at that time.

With the advent of Run 2, a large sample of $p p$ collisions at $\sqrt{s}=5.02 \mathrm{TeV}$ was provided by the LHC enabling the study of inclusive charged-particle production in $p p$ collisions. ATLAS has measured chargedparticle spectra in the $p p$ system at $\sqrt{s}=5.02 \mathrm{TeV}$ using an integrated luminosity of $25 \mathrm{pb}^{-1}$. Then a nuclear modification factor, $R_{p \mathrm{~Pb}}$, is extracted from charged-particle spectra measured for $p+\mathrm{Pb}$ collisions in Ref. [10] for a very wide range of transverse momentum $4<p_{\mathrm{T}}<189 \mathrm{GeV}$ and rapidity $-2.0<y<1.5$.

Figure 4 shows $R_{p \mathrm{~Pb}}$ in the $0-90 \%$ centrality interval. The $p_{\mathrm{T}}$ dependence is substantially reduced compared 170 to the previous estimate from ATLAS that relied upon 171 interpolation. No significant deviation from unity is ob- 172 served in the centrality interval $0-90 \%$ for any trans- 173 verse momentum.

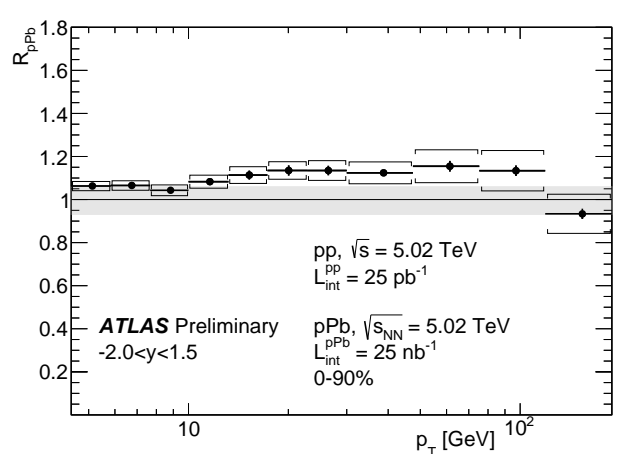

Figure 4: Nuclear modification factor $R_{p \mathrm{~Pb}}$ for charged particles measured in the $0-90 \%$ centrality interval [8]. Statistical uncertainties are shown with vertical lines and brackets represent systematic uncertainties, with the exception of the fully correlated systematic uncertainty that is shown with the gray band around unity.

143 144 145

ATLAS has reported an evidence for light-bylight (LbyL) scattering signatures in quasi-real photon interactions occurring in ultra-peripheral $\mathrm{Pb}+\mathrm{Pb}$ collisions at $\sqrt{s_{\mathrm{NN}}}=5.02 \mathrm{TeV}$ [13]. The studied data set 174 corresponds to $480 \mu \mathrm{b}^{-1}$. After applying event selection 


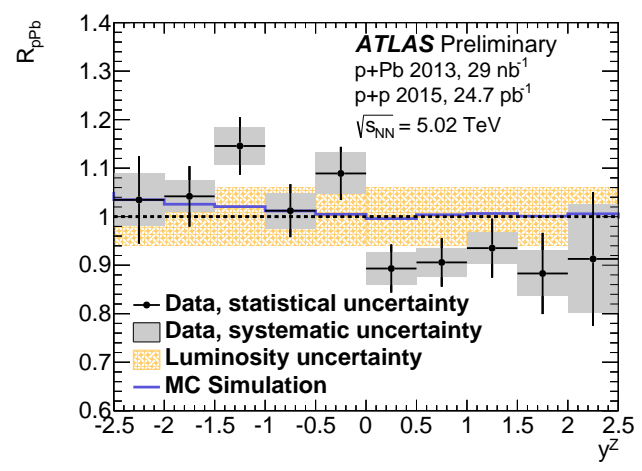

Figure 5: Nuclear modification factor as a function of $Z$ boson rapidity [11]. The uncertainties on each point include statistical and systematic uncertainties from $p+\mathrm{Pb}$ and $p p$ measurements. The band around unity represents the uncertainty of the luminosities. The blue line shows an expected $R_{p} \mathrm{~Pb}$ based on simulation.

criteria requiring no activity in the detecor except for two well reconstructed photons, 13 event are observed, while 7.3 signal events are expected from predictions. The dominant background processes, i.e. central exclusive production $g g \rightarrow \gamma \gamma$, exclusive dilepton production $\gamma \gamma \rightarrow e^{+} e^{-}$as well as other fake-photon backgrounds, are estimated using data-driven methods. After accounting for all of these sources, 2.6 background events are expected, with an uncertainty of 0.7 . Figure 6 shows an acoplanarity ${ }^{1}$ distribution for the diphoton candidates with $p_{\mathrm{T}}^{\gamma \gamma}<2 \mathrm{GeV}$. A good agreement between a sum of signal plus background contributions and the data is observed. The significance is determined to be $4.4 \sigma$. After background subtraction and corrections, the cross section of the $\gamma \gamma \rightarrow \gamma \gamma$ process for photon transverse momentum, $E_{\mathrm{T}}>3 \mathrm{GeV}$, photon pseudorapidity, $|\eta|<2.4$, invariant mass of the diphoton system, $m^{\gamma \gamma}>6 \mathrm{GeV}$, diphoton transverse momentum, $p_{\mathrm{T}}^{\gamma \gamma}<2 \mathrm{GeV}$ and acoplanarity, Aco $<0.01$ is measured to be $70 \pm 20$ (stat.) \pm 17 (syst.) nb, which is compatible with the Standard Model prediction of $49 \pm 10 \mathrm{nb}$ [14].

\section{Particle correlations}

\section{Flow harmonics in $\mathrm{Pb}+\mathrm{Pb}$ collisions}

Azimuthal anisotropy of charged particles in $\mathrm{Pb}+\mathrm{Pb}$ collisions at $\sqrt{s_{\mathrm{NN}}}=5.02 \mathrm{TeV}$ has been measured using Scalar Product (SP), Event Plane (EP) and Two Particle Correlations (2PC) methods [15]. The measurements are done using an integrated luminosity of

\footnotetext{
${ }^{1}$ Acoplanarity of the diphoton system is defined as Aco $=1-\frac{\Delta \phi_{\gamma \gamma}}{\pi}, \quad{ }^{235}$ where $\Delta \phi_{\gamma \gamma}$ is a difference in azimuthal angles of two photons. $\quad 236$
}

Figure 6: Diphoton acoplanarity distribution for events in the LbyL signal region [13].
204
$5 \mu \mathrm{b}^{-1}$ in SP/EP and $22 \mu \mathrm{b}^{-1}$ in $2 \mathrm{PC}$ results. The azimuthal anisotropies, quantified by the flow harmonics $v_{\mathrm{n}}$, are measured using the $\mathrm{SP}(n=2-7)$, EP $(n=$ $2-5)$ and 2PC $(n=1-6)$ methods. The measurements are done over wide transverse momentum $(0.5<$ $\left.p_{\mathrm{T}}<25 \mathrm{GeV}\right)$, pseudorapidity $(|\eta|<2.5)$ and centrality $(0-80 \%)$ ranges. All harmonics show similar $p_{\mathrm{T}^{-}}$ dependence, first increasing with $p_{\mathrm{T}}$ up to a maximum at around $3-4 \mathrm{GeV}$ and then decreasing for higher $p_{\mathrm{T}}$ values. However, significant values of the second order harmonic $v_{2}$ persist up to $25 \mathrm{GeV}$. Figure 7 shows $v_{\mathrm{n}}$ as a function of event centrality for the SP method. The elliptic flow signal is strongly dependent on event centrality and it is largest in mid-central events (50-60\%). The higher-order harmonics show a weak centrality dependence, which is consistent with an anisotropy associated with fluctuations in the initial geometry. An inset shows the $v_{6}$ and $v_{7}$ harmonics with adjusted scale. The latter could be measured for the first time in $\mathrm{Pb}+\mathrm{Pb}$ collisions.

A hierarchy $v_{\mathrm{n}+1}<v_{\mathrm{n}}$ can be observed for harmonics from 3 to 7 for all ranges of $p_{\mathrm{T}}$ and all centralities. The $v_{\mathrm{n}}$ coefficients are found to exhibit only a weak $\eta$ dependence across all centrality intervals. The results obtained using the EP and SP methods are consistent for harmonics of order $n \geq 3$. A small, systematic difference is observed for $v_{2}$, where the values obtained from the SP method are up to 3\% larger than the values obtained using the EP method. The 2PC and SP methods give values for $v_{\mathrm{n}}$ that are quite consistent up to $10 \mathrm{GeV}$. However, in the most central events the SP method gives systematically larger values for $v_{2}$ for $p_{\mathrm{T}}>2 \mathrm{GeV}$. Comparisons to measurements in $\mathrm{Pb}+\mathrm{Pb}$ collisions at $2.76 \mathrm{TeV}$ show that the $p_{\mathrm{T}}$ dependence of the $v_{\mathrm{n}}$ shows no change between 2.76 and $5.02 \mathrm{TeV}$. 


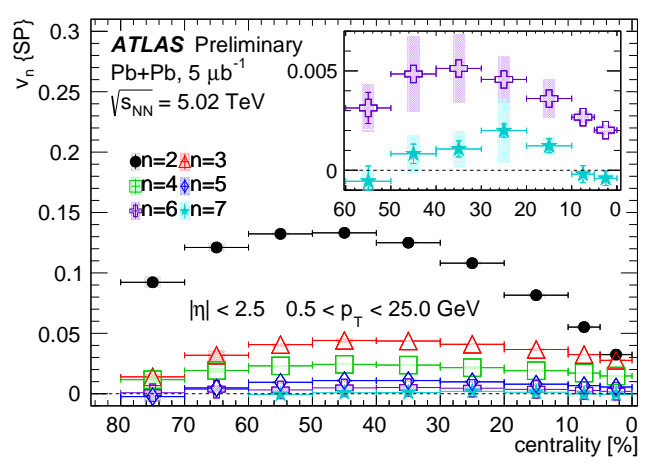

Figure 7: Integrated $v_{\mathrm{n}}\{\mathrm{SP}\}$ as a function of centrality integrated over $|\eta|<2.5$ and $0.5<p_{\mathrm{T}}<25 \mathrm{GeV}$ extracted from the SP method [15]. In the inset the $v_{6}$ and $v_{7}$ are shown with adjusted scale. Results are averaged over the intervals indicated by horizontal error bars. The vertical error bars indicate statistical uncertainties. The shaded areas indicate systematic uncertainties.

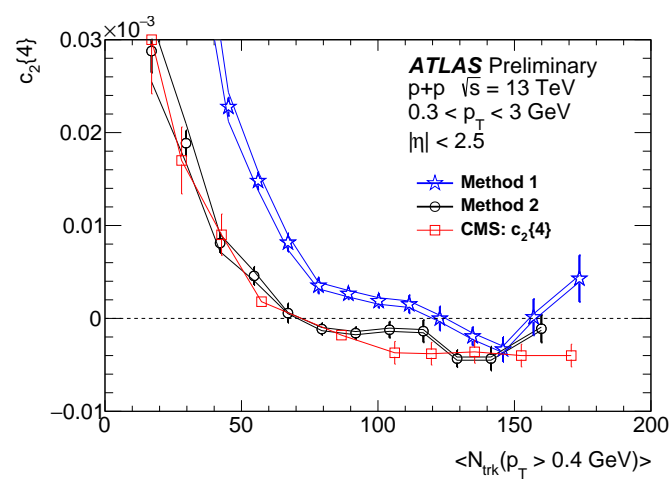

Figure 8: Comparison of $c_{2}\{4\}$ cumulants obtained with Method 1 and Method 2 for $p p$ collisions at $13 \mathrm{TeV}$ [16]. The plot shows results for reference particles with $0.3<p_{\mathrm{T}}<3.0 \mathrm{GeV}$. The error bars and shaded bands denote statistical and systematic uncertainties, respectively. The CMS results from Ref. [17] are also shown as open squares.

\section{Azimuthal anisotropies in $p p$ and $p+P b$ systems}

Two-charged-particle correlations have been measured by ATLAS in $p p$ collisions at 5.02 and $13 \mathrm{TeV}$ and in $5.02 \mathrm{TeV} p+\mathrm{Pb}$ collisions [18]. This measurement uses integrated luminosities of $64 \mathrm{nb}^{-1}$ for the $13 \mathrm{TeV}$ $p p$ data, $170 \mathrm{nb}^{-1}$ for $5.02 \mathrm{TeV} p p$ data and $28 \mathrm{nb}^{-1}$ for $5.02 \mathrm{TeV} p+\mathrm{Pb}$ data. A template fitting procedure developed for $p p$ collisions [19] is utilised systematically in all data sets. The correlation functions are measured for different intervals of measured charged particle multiplicity $\left(N_{\mathrm{ch}}^{\mathrm{rec}}\right)$ and FCal transverse energy and for different intervals of charged-particle transverse momentum; many of the results are presented for an inclusive $p_{\mathrm{T}}$ interval of $0.5<p_{\mathrm{T}}<5 \mathrm{GeV}$.

In addition to the elliptic, $\cos (2 \Delta \phi)$, modulation observed in a previous measurement, the $p p$ correlation functions are found to exhibit significant $\cos (3 \Delta \phi)$ and $\cos (4 \Delta \phi)$ modulation. The Fourier coefficients $v_{\mathrm{n}, \mathrm{n}}$ associated with the $\cos (n \Delta \phi)$ modulation of the correlation functions for $n=2-4$ are measured as a function of charged-particle multiplicity and charged-particle transverse momentum. Amplitudes of flow harmonics are found to have a similar $p_{\mathrm{T}}$ dependence in both systems, however, they they exhibit a systematically different magnitude.

A direct test of the similarity of the $p_{\mathrm{T}}$ dependence of the Fourier coefficients in $p p$ and $p+\mathrm{Pb}$ collisions is provided in Figure 9 for $n=2$. The $p p v_{2}$ values have been multiplied by 1.51 to match the $v_{2}\left(p_{\mathrm{T}}^{\mathrm{a}}\right)$ function at maximum. The resulting $v_{2}\left(p_{\mathrm{T}}^{\mathrm{a}}\right)$ values for (scaled) $p p$ and $p+\mathrm{Pb}$ agree well for $p_{\mathrm{T}}^{\mathrm{a}}$ up to $5 \mathrm{GeV}$. At higher $p_{\mathrm{T}}^{\mathrm{a}}$ 
the $p p v_{2}$ decreases more rapidly due to the multiplicity- 317 dependent change in the shape of the dijet peak in the two-particle correlation function at high $p_{\mathrm{T}}$. After the ${ }^{318}$ scaling, the $p p v_{2}\left(p_{\mathrm{T}}^{\mathrm{a}}\right)$ are slightly higher than the $p+\mathrm{Pb}{ }^{319}$ at low $p_{\mathrm{T}}^{\mathrm{a}}$, but the similarity of the shapes of the $p_{\mathrm{T}}$ de- ${ }^{320}$ pendence is, nonetheless, striking.

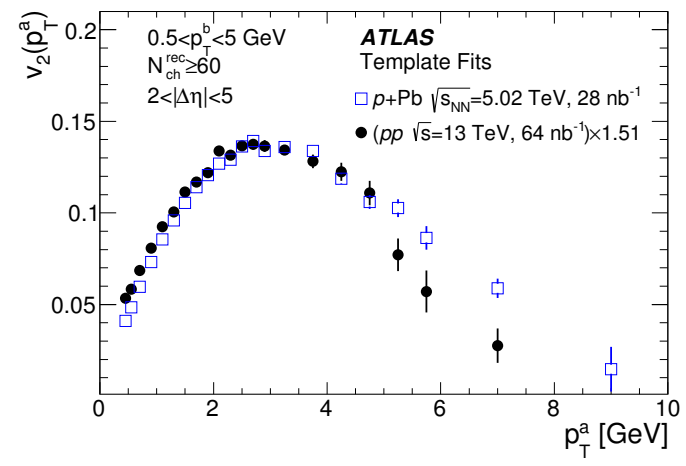

Figure 9. Comparison of the shapes of the $v_{2}\left(p_{\mathrm{T}}\right)$ in the $13 \mathrm{TeV} p p$ and $5.02 \mathrm{TeV} p+\mathrm{Pb}$ data [18]. The $p p v_{2}$ has been scaled by a factor of 1.51 along the $y$-axis in order to match the maximum of the $v_{2}$ in the two data sets. The results are for $0.5<p_{\mathrm{T}}^{\mathrm{b}}<5 \mathrm{GeV}$ and $N_{\mathrm{ch}}^{\mathrm{rec}} \geq 60$. The error bars indicate statistical uncertainties.

Figure 10 shows $v_{4} / v_{2}^{2}$ versus $N_{\mathrm{ch}}^{\text {rec }}$ for the $13 \mathrm{TeV} p p$ and $p+\mathrm{Pb}$ data. In the ratio, the correlated systematic ${ }_{342}$ uncertainties between the measured $v_{4}$ and $v_{2}^{2}$ cancel. The ratio is observed to be constant as a function of $N_{\mathrm{ch}}^{\mathrm{rec}}$ for both data sets even though the $p+\mathrm{Pb} v_{2}$ and $v_{4}$ increase with $N_{\mathrm{ch}}^{\text {rec }}$. The $v_{4} / v_{2}^{2}$ ratio is observed to be $50 \%$ larger in the $p p$ data than in the $p+\mathrm{Pb}$ data. Naively, this 345 would indicate a larger non-linear contribution to $v_{4}$ in ${ }^{346}$ $p p$ collisions compared to $p+\mathrm{Pb}$ collisions.

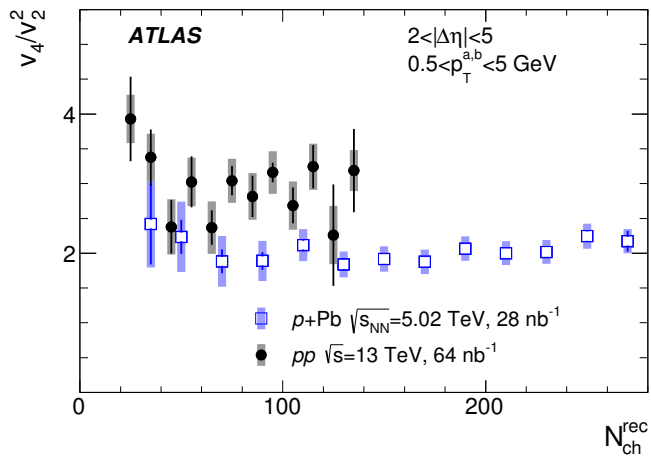

Figure 10: Ratio of $v_{4}$ to $v_{2}^{2}$ as a function of $N_{\mathrm{ch}}^{\mathrm{rec}}$ in the $13 \mathrm{TeV} p p$ and 366 $5.02 \mathrm{TeV} p+\mathrm{Pb}$ data [18]. The results are for $0.5<p_{\mathrm{T}}^{\mathrm{a}, \mathrm{b}}<5 \mathrm{GeV}$. ${ }^{367}$ The error bars and shaded bands indicate statistical and systematic uncertainties, respectively.

\section{Summary}

The ATLAS Collaboration has performed multiple measurements on heavy-ion data collected at the center-of-mass energy $\sqrt{s_{\mathrm{NN}}}=5.02 \mathrm{TeV}$ provided in the heavy-ion run in 2015. They involve soft and hard probes and aim to elucidate the short- and longwavelength properties of the QGP. Results of azimuthal and multi-particle correlations in both $p p$ and $\mathrm{Pb}+\mathrm{Pb}$ systems have been shown as examples of soft-sector measurements. An impact of the QGP medium on quarkonia and jets have been discussed in the context of $J / \psi$ and $\psi(2 S)$ production as well as photon-jet correlations. Availability of $p p$ data collected at the same energy as the $\mathrm{Pb}+\mathrm{Pb}$ system allows for direct comparisons of various observables between two collision systems. In addition, old Run $1 p+\mathrm{Pb}$ data collected at $\sqrt{s_{\mathrm{NN}}}=5.02 \mathrm{TeV}$ could be reinterpreted thanks to the new $p p$ reference sample. That allows for a better precision in measurement sensitive to nuclear modifications to parton distribution functions. Furthermore, larger luminosity and energy available in Run 2 makes it possible to provide an evidence of light-by-light scattering in $\mathrm{Pb}+\mathrm{Pb}$ collisions for the first time.

This work was supported in part by the National Science Center, Poland grant DEC-2015/18/M/ST2/00087, and by PL-Grid Infrastructure.

\section{References}

[1] STAR Collaboration, J. Adams, et al., Nucl. Phys. A757, 102 (2005)

[2] BRAHMS Collaboration, I. Arsene, et al., Nucl. Phys. A757, 1 (2005)

[3] PHOBOS Collaboration, B.B. Back, et al., Nucl. Phys. A757, 28 (2005)

[4] PHENIX Collaboration, K. Adcox et al., Nucl. Phys. A757, 184 (2005),

[5] ATLAS Collaboration, JINST 3 (2008) 03

[6] ATLAS Collaboration, ATLAS-CONF-2016-109

[7] ATLAS Collaboration, ATLAS-CONF-2016-110

[8] ATLAS Collaboration, ATLAS-CONF-2016-108

[9] CMS Collaboration, Eur. Phys. J. C75 (2015) 237

[10] ATLAS Collaboration, Phys. Lett. B763 (2016) 313

[11] ATLAS Collaboration, ATLAS-CONF-2016-107

[12] ATLAS Collaboration, Phys. Rev. C92 (2015) 044915

[13] ATLAS Collaboration, ATLAS-CONF-2016-111

[14] M. Klusek-Gawenda, P. Lebiedowicz and A. Szczurek, Phys. Rev. C93 (2016) 044907

[15] ATLAS Collaboration, ATLAS-CONF-2016-105

[16] ATLAS Collaboration, ATLAS-CONF-2016-106

[17] CMS Collaboration, arXiv: 1606.06198 [nucl-ex]

[18] ATLAS Collaboration, arXiv: 1609.06213 [nucl-ex]

[19] ATLAS Collaboration, Phys. Rev. Lett. 116 (2016) 172301 